\title{
Validación de la metodología analítica para determinar la cuantificación de taninos en la pepa de palta (Persea americana Miller var. hass) por cromatografía líquida de alta presión
}

\begin{abstract}
RESUMEN
En el presente trabajo de investigación intitulado Validación de una metodología analítica para determinar Taninos (Catequina) en pepa de palta (Persea americana Miller var. hass) por cromatografia liquida de alta resolución" (HPLC), tiene por objetivo validar una metodología por HPLC para determinar la concentración de estos analitos, haciendo uso de un cromatógrafo Agilent 1100 con arreglo de diodos, se utilizó un detector UV-Visible con una columna Octadecilsilano (C18) de $150 \mathrm{~mm}$ de longitud y 4,6 $\mathrm{mm}$ de diámetro interior; como fase móvil se empleó una solución $\mathrm{A}$ (metanol) y una solución $\mathrm{B}$ (ácido acético glacial al $0,1 \%$ ), con un tiempo de recorrido de 15 minutos, en una gradiente isocrática de $15 \%$ de Ay $85 \%$ de B; leyéndose a una longitud de onda de $210 \mathrm{~nm}$, con una velocidad de flujo de $1 \mathrm{~mL} / \mathrm{min}$., se inyectó una muestra de $10 \mu \mathrm{L}$, a una temperatura de $35^{\circ} \mathrm{C}$. Encontrándose que los parámetros evaluados: linealidad, límite de detección, límite de cuantificación, precisión del método y exactitud, están dentro de los valores aceptados para una validación.
\end{abstract}

Palabras clave: validación, taninos (catequina), HPLC

VALIDATION OF ANALYTICAL METHODOLOGY TO DETERMINE THE QUANTIFICATION OF TANNINS IN THE PIT AVOCADo (PERSEA AMERICANA MILLER VAR. HASS) BY HIGH PRESSURE LIQUID CHROMATOGRAPHY

\section{ABSTRACT}

In the present research work entitled Validation of an analytical methodology for determining tannins (catechin) in pit (integument) avocado (Persea americana Miller var. Hass) by high resolution liquid chromatography "(HPLC), is to validate a methodology HPLC to determine the concentration of these analytes, using an Agilent 1100 gas chromatograph with diode array detector was used with a UV-Visible octadecylsilane column (C18) of $150 \mathrm{~mm}$ length and $4.6 \mathrm{~mm}$ internal diameter; was used as mobile phase solution A (methanol) and solution B (glacial acetic acid $0.1 \%$ ), with a travel time of 15 minutes, in an isocratic gradient of $15 \%$ A and $85 \%$ B; read at a wavelength of $210 \mathrm{~nm}$, with a flow rate of $1 \mathrm{~mL} / \mathrm{min}$. was injected 10 $\mathrm{uL}$ sample at a temperature of $35^{\circ} \mathrm{C}$. Finding that the evaluated parameters: linearity, limit of detection, limit of quantification, precision and accuracy of the method are within accepted values for validation.

Keywords: validation, tannins (catechin), HPLC

\section{INTRODUCCIÓN}

En las últimas décadas los fitofármacos han ido ganando terreno dentro del arsenal terapéutico mundial, fundamentalmente por su escasa toxicidad, bajos costos y por utilizar tecnologías de bajos niveles de inversión e insumos. Lo cual a su vez ha incidido poderosamente en la expansión, desarrollo y consolidación de la producción de dichos medicamentos. Se dice que el $80 \%$ de la población mundial utiliza plantas para el tratamiento de las enfermedades y en los países industrializados el $35 \%$ de los medicamentos prescritos contienen principios activos de origen natural ${ }^{1}$.Todo lo anterior da una medida de la importancia de los fitofármacos para los países del tercer mundo; por su parte en Cuba se le ha dado una importancia estratégica a los mismos y se ha ido desarrollando todo un sistema que abarca desde la agrotecnia de la especie vegetal (palta), hasta la confección del producto terminado y su registro.

La parte activa de dicho producto es el extracto acuoso de la pepa (Persea Americana Millar var. hass).

El presente trabajo tiene como objetivo la validación de un método analítico en HPLC de alta resolución para la cuantificación de los taninos el mismo, que sustituya al método volumétrico actualmente en uso, el cual no es preciso, tiene una alta dependencia del analista y es muy laborioso.

\section{MATERIALES Y MÉTODOS}

\subsection{Métodos}

Las muestras de pepa de palta fueron adquiridas en el centro de acopio (mercado mayorista de Trujillo, proveniente de la sierra del departamento de La Libertad).

Se preparó una solución madre de Catequina de $1 \mathrm{mg} / \mathrm{mL}$, de la cual se tomó $10,50,100,150,200$ y $250 \mu \mathrm{L}$ y se les adicionó a cada una de ellas en fiolas de $10 \mathrm{~mL}$, llevándose a su respectivo aforo con agua ultrapura. De la cual se obtuvieron soluciones de $1,5,10,15,20$ y $25 \mu \mathrm{g} / \mathrm{mL}$ respectivamente.

\footnotetext{
Profesor investigador de la Facultad de Ingeniería, Escuela de Ingeniería Agroindustrial de la Universidad Nacional del Santa.

** Profesor investigador de la Facultad de Ingeniería Química de la Universidad Nacional de Trujillo.
} 
Se pesó $0,5 \mathrm{~g}$ de polvo de tegumento de la pepa de palta y se le colocó en un tubo de ensayo, se le agregó $4 \mathrm{~mL}$ de agua ultra pura y se llevó al ultrasonido por 15 minutos, luego se centrifugo a 3500 rpm por un lapso de 10 minutos, se tomó 2 $\mathrm{mL}$ del sobrenadante y se filtró haciendo uso de filtros jeringa de celulosa regenerada de $25 \mathrm{~mm}$ de diámetro y $0,2 \mu \mathrm{m}$ de poro; colocándose en viales ámbar de 1,5 mL.

Para determinar la linealidad del método, se evaluaron 6 soluciones estándares comprendidas entre 1 y $25 \mu \mathrm{g} / \mathrm{mL}$ de Catequina empleándose un cromatógrafo Agilent 1100 con arreglo de diodos, se utilizó un detector UV-Visible con una columna Octadecilsilano (C18) de $150 \mathrm{~mm}$ de longitud y 4,6 mm de diámetro interior; como fase móvil se empleó una solución $A$ (metanol) y una solución $B$ (ácido acético glacial al 0,1\%), con un tiempo de recorrido de 15 minutos, en una gradiente isocrática de $15 \%$ de $A$ y $85 \%$ de $B$; leyéndose a una longitud de onda de $210 \mathrm{~nm}$, con una velocidad de flujo de $1 \mathrm{~mL} / \mathrm{min}$., se inyectó una muestra de $10 \mu \mathrm{L}$, a una temperatura de $35^{\circ} \mathrm{C}$.

Se preparó soluciones estándares de Catequina menoresa $1 \mu \mathrm{g} / \mathrm{mL}$, hasta encontrarlaconcentración, a la cual en el cromatograma aparezca un pico en el tiempo de retención característico del analito, pero que no sea cuantificable por el equipo.

Se obtuvo aplicando la fórmula:

$$
\text { L.C. }=(10 / 3) \text { L.D. }
$$

\section{Donde:}

L.C.: Límite de cuantificación.

L.D.: Límite de detección.

Para determinar la precisión del método, se evaluó mediante nueve determinaciones con tres niveles de concentración. Se prepararon muestras de $8 \mu \mathrm{g} /$ $\mathrm{mL}, 10 \mu \mathrm{g} / \mathrm{mL}$ y $12 \mu \mathrm{g} / \mathrm{mL}$ de Catequina por triplicado, y se analizaron mediante un cromatógrafo Agilent 1100 con arreglo de diodos, se utilizó un detector UV-Visible con una columna Octadecilsilano (C18) de $150 \mathrm{~mm}$ de longitud y $4,6 \mathrm{~mm}$ de diámetro interior; como fase móvil se empleó una solución $A$ (metanol) y una solución B (ácido acético glacial al $0,1 \%$ ), con un tiempo de recorrido de 15 minutos, en una gradiente isocrática de $15 \%$ de $\mathrm{A}$ y $85 \%$ de $B$; leyéndose a una longitud de onda de $210 \mathrm{~nm}$, con una velocidad de flujo de $1 \mathrm{~mL} / \mathrm{min}$., se inyectó una muestra de $10 \mu \mathrm{L}$, a una temperatura de $35^{\circ} \mathrm{C}$.

La exactitud del método se evaluó mediante nueve determinaciones con tres niveles de concentración. Se añadieron cantidades conocidas de Catequina a la muestra problema analizada previamente para obtener concentraciones finales de $10 \mu \mathrm{g} / \mathrm{mL}, 12$ $\mu \mathrm{g} / \mathrm{mL}$ y $14 \mu \mathrm{g} / \mathrm{mL}$ por triplicado y se analizaron mediante un cromatógrafo Agilent 1100 con arreglo de diodos, se utilizó un detector UV-Visible con una columna Octadecilsilano (C18) de $150 \mathrm{~mm}$ de longitud y 4,6 $\mathrm{mm}$ de diámetro interior; como fase móvil se empleó una solución $A$ (metanol) y una solución B (ácido acético al $0,1 \%$ ), con un tiempo de recorrido de 15 minutos, en una gradiente isocrática de $15 \%$ de $A$ y $85 \%$ de $B$; leyéndose a una longitud de onda de $210 \mathrm{~nm}$, con una velocidad de flujo de $1 \mathrm{~mL} / \mathrm{min}$., se inyectó una muestra de 10 $\mu \mathrm{L}$, a una temperatura de $35^{\circ} \mathrm{C}$.

\subsection{Materiales}

Material de estudio: se utilizó un estándar de Taninos (catequina) en el tegumento y muestras de pepa de palta hass.

Material de vidrio: fiolas de 25, 50, 100, 500 y 1000 $\mathrm{mL}$, matraces Erlenmeyer de 250 y $500 \mathrm{~m}$, pipetas graduadas de $1,2,5$ y $10 \mathrm{~m}$, probetas de 25 y 100 $\mathrm{m}$, vasos de precipitación de 50, 100, 500 y $1000 \mathrm{~m}$, viales ámbar de $1,5 \mathrm{~mL}$.

Reactivos: ácido acético glacial, agua destilada, agua ultra pura, catequina y metanol grado HPLC

Equipos: balanza Analítica Sartorius 2942, Sensibilidad $0,0001 \mathrm{~g}$, cromatógrafo Agilent 1100 series, filtro de vacío SANAILAB, micropipeta 100-1000 uL, micropipeta 20-200 uL y sistema de purificación para obtener agua ultra pura: Modelo: EASY PURE, marca: Barnsted

\section{RESULTADOS Y DISCUSIONES}

\subsection{Resultados}

Tabla 1. Linealidad de las concentraciones en $\mathrm{mg} /$ $\mathrm{mL}$ de Taninos (Catequina)

\begin{tabular}{|c|c|c|}
\hline Nivel & $\begin{array}{c}\text { Concentración } \\
\mathbf{m g} / \mathbf{m L}\end{array}$ & Área pico (mUA*s) \\
\hline 1 & 1 & 32,13851 \\
\hline 2 & 5 & 180,08177 \\
\hline 3 & 10 & 375,92230 \\
\hline 4 & 15 & 572,80670 \\
\hline 5 & 20 & 767,45276 \\
\hline 6 & 25 & 947,67609 \\
\hline
\end{tabular}


Gráfico 1. Curva de calibración de Taninos (Catequina).

\section{CURVA DE CALIBRACIÓN DE CATEQUINA}

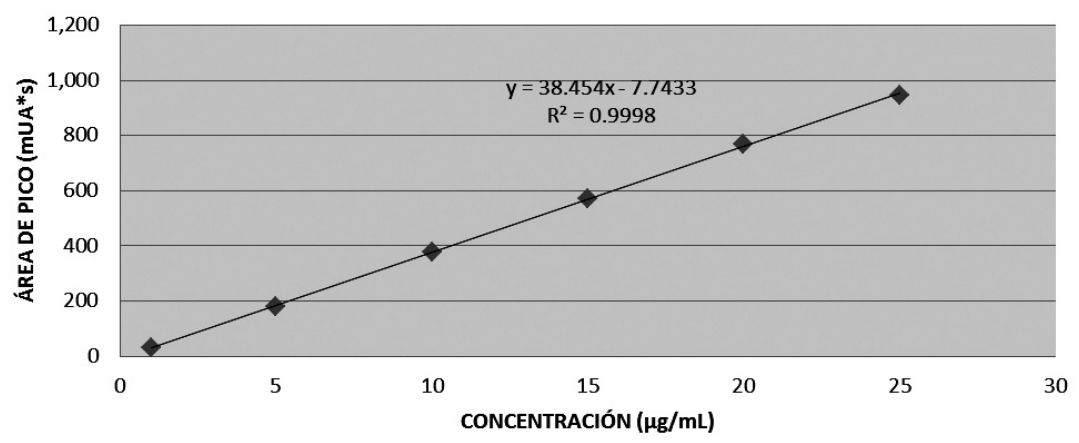

Tabla 2. Límite de detección de Taninos (Catequina)

\begin{tabular}{|c|c|c|}
\hline ANALITO & CONCENTRACIÓN $(\boldsymbol{\mu g} / \mathrm{mL})$ & ÁREA PICO $\left(\mathbf{m U A}^{*} \mathbf{s}\right)$ \\
\hline Catequina & 0,21 & 0,33204 \\
\hline
\end{tabular}

Tabla 3. Límite de cuantificación de Taninos (Catequina)

\begin{tabular}{|c|c|c|}
\hline ANALITO & CONCENTRACIÓN $(\mu \mathrm{g} / \mathrm{mL})$ & ÁREA PICO $\left(\mathrm{mUA}^{*} \mathbf{s}\right)$ \\
\hline Catequina & 0,7 & 30,71070 \\
\hline
\end{tabular}

Tabla 4. Precisión del método en la determinación de Taninos (Catequina)

\begin{tabular}{|c|c|c|c|c|c|c|c|}
\hline Ensayo & $\begin{array}{c}\text { Concentración } \\
(\mathrm{mg} / \mathrm{mL})\end{array}$ & $\begin{array}{c}\text { Área pico } \\
\left(m^{*} A^{*} \mathbf{s}\right)\end{array}$ & $\begin{array}{l}\text { Cantidad } \\
\text { recuperada } \\
(\mathrm{mg} / \mathrm{mL})\end{array}$ & $\begin{array}{c}\text { Cantidad } \\
\text { recuperada (\%) } \\
(98,0 \%-102,0 \%)\end{array}$ & Promedio (\%) & $\begin{array}{l}\text { Desviación } \\
\text { estándar }\end{array}$ & $\begin{array}{c}\text { Desviación } \\
\text { estándar } \\
\text { relativa (\%) } \\
(2,0 \%)\end{array}$ \\
\hline \multirow{3}{*}{1} & \multirow{3}{*}{$8(80 \%)$} & 294,70282 & 7,86 & 98,31 & \multirow{3}{*}{99,71} & \multirow{3}{*}{1,76} & \multirow{3}{*}{1,77} \\
\hline & & 305,12030 & 8,13 & 101,70 & & & \\
\hline & & 297,24002 & 7,93 & 99,13 & & & \\
\hline \multirow{3}{*}{2} & \multirow{3}{*}{$10(100 \%)$} & 376,56897 & 9,99 & 99,94 & \multirow{3}{*}{100,36} & \multirow{3}{*}{1,25} & \multirow{3}{*}{1,24} \\
\hline & & 383,63437 & 10,17 & 101,77 & & & \\
\hline & & 374,43006 & 9,93 & 99,38 & & & \\
\hline \multirow{3}{*}{3} & \multirow{3}{*}{$12(120 \%)$} & 452,04816 & 11,95 & 99,64 & \multirow{3}{*}{99,83} & \multirow{3}{*}{0,89} & \multirow{3}{*}{0,89} \\
\hline & & 449,36517 & 11,88 & 99,05 & & & \\
\hline & & 457,43646 & 12,09 & 100,80 & & & \\
\hline \multicolumn{4}{|c|}{ PROMEDIO (\%) } & 99,97 & & & \\
\hline \multicolumn{4}{|c|}{ DESVIACIÓN ESTÁNDAR } & 1,20 & & & \\
\hline \multicolumn{4}{|c|}{ DESVIACIÓN ESTÁNDAR RELATIVA (\%) $(2,0 \%)$} & 1,20 & & & \\
\hline
\end{tabular}




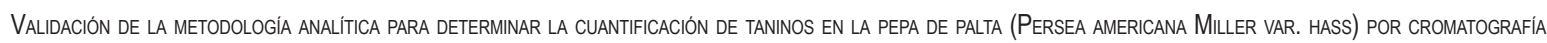

Tabla 5. Exactitud del método en la determinación de Catequina en pepa de palta

\begin{tabular}{|c|c|c|c|c|c|c|c|}
\hline Ensayo & $\begin{array}{l}\text { Concentración } \\
(\mathrm{mg} / \mathrm{mL})\end{array}$ & $\begin{array}{l}\text { Área pico } \\
\left(m U A^{*} s\right)\end{array}$ & $\begin{array}{l}\text { Cantidad } \\
\text { recuperada } \\
(\mathrm{mg} / \mathrm{mL})\end{array}$ & \begin{tabular}{|c} 
Cantidad \\
recuperada (\%) \\
$(98,0 \%-102,0 \%)$
\end{tabular} & Promedio (\%) & $\begin{array}{l}\text { Desviación } \\
\text { estándar }\end{array}$ & $\begin{array}{c}\text { Desviación } \\
\text { estándar } \\
\text { relativa (\%) } \\
(2,0 \%)\end{array}$ \\
\hline \multirow{3}{*}{1} & \multirow{3}{*}{10} & 377,72824 & 10,02 & 100,24 & \multirow{3}{*}{99,55} & \multirow{3}{*}{0,66} & \multirow{3}{*}{0,66} \\
\hline & & 372,66486 & 9,89 & 98,92 & & & \\
\hline & & 374,83847 & 9,94 & 99,49 & & & \\
\hline \multirow{3}{*}{2} & \multirow{3}{*}{12} & 460,75049 & 12,18 & 101,52 & \multirow{3}{*}{100,78} & \multirow{3}{*}{0,66} & \multirow{3}{*}{0,65} \\
\hline & & 454,89499 & 12,03 & 100,25 & & & \\
\hline & & 456,27179 & 12,06 & 100,55 & & & \\
\hline \multirow{3}{*}{3} & \multirow{3}{*}{14} & 538,66953 & 14,20 & 101,49 & \multirow{3}{*}{101,07} & \multirow{3}{*}{0,76} & \multirow{3}{*}{0,75} \\
\hline & & 531,64719 & 14,02 & 100,19 & & & \\
\hline & & 538,81268 & 14,21 & 101,52 & & & \\
\hline \multicolumn{4}{|c|}{ PROMEDIO (\%) } & 100,46 & & & \\
\hline \multicolumn{4}{|c|}{ DESVIACIÓN ESTÁNDAR } & 0,92 & & & \\
\hline \multicolumn{4}{|c|}{ DESVIACIÓN ESTÁNDAR RELATIVA (\%) $(2,0 \%)$} & 0,91 & & & \\
\hline
\end{tabular}

\section{CONCLUSIONES}

De los resultados obtenidos se puede concluir en:

- La curva de calibración para Taninos (Catequina) está regida por la ecuación $Y=38,454 X-7,7433$ con un coeficiente de correlación de 0,9998.

- El límite de detección para Taninos (Catequina) es de $0,21 \mu \mathrm{g} / \mathrm{mL}$.

- El límite de cuantificación para Taninos (Catequina) es de $0,7 \mu \mathrm{g} / \mathrm{mL}$.

- La precisión del sistema en la determinación de Taninos (Catequina) obtuvo una desviación estándar relativa menor al $2 \%$.

- La precisión del método en la determinación de Taninos (Catequina) obtuvo una desviación estándar relativa menor al $2 \%$.

- La exactitud del método en la determinación de Taninos (Catequina), obtuvo una desviación estándar relativa menor al $2 \%$.

\section{LITERATURA CITADA}

[1] Contreras-Domínguez M, Marnet N, PerraudGaime I, Roussos S, Guyot S, Augur C. Degradación inicial de taninos condensados por Aspergillus fumigatus MC8. XI Congreso
Nacional De Biotecnología y Bioingeniería. 2005; México (Yucatán).

[2] Sánchez LM, Mancebo B, Faure R, Travieso MC. Validación de la técnica para la determinación de catequina en tabletas de Rhizophora mangle L. por CLAR. Revista Cubana de Farmacia. 2010; 45(1): 58-70.

[3] Cala M, Vásquez A, Garcia A, Martinez JR, Stashenko E. Estudio comparativo por electroforesis capilar y cromatografía líquida de alta eficiencia de catequinas extraídas de cinco variedades de cacao colombiano. Rev Acad Colomb Cienc. 2011; 136(35): 371-379.

[4] Gracia Nava Manuel Alejandro. Cuantificación de fenoles y flavonoides totales en extractos naturales. Universidad Autónoma de Querétaro. Rev Acad 2009; 1 -4.

[5] Mónica Cala, Ángela Vásquez, Alejandro García. Estudio comparativo por electroforesis capilar y cromatografía líquida de alta eficiencia de catequinas extraídas de cinco variedades de cacao colombiano. Rev. Acad. Colomb. Cienc.: Volumen xxxv, número 136-septiembre de 2011: 2 -3.

[6] J. A. García-Fajardo; M. del R. Ramos-Godínez; J. Mora-Galindo Estructura de la semilla de 
aguacate y cuantificación de la grasa extraída por diferentes técnicas 1999. Revista Chapingo Serie Horticultura 5: 123-128. 3-4.

[7] Miguel A. Sogorb Sánchez, Eugenio Vilanova Gisbert. 2004. "Técnicas Analíticas de Contaminantes Químicos" Aplicaciones Toxicológicas, Medioambientales y Alimentarias. Editorial Dios de Santos - España. Cap. 9, pág. 173.

[7] Ramón Compañó B.- Ángel Ríos C. "Garantía de la Calidad en los Laboratorios Analíticos". Editorial Síntesis - España. Cap. 11, pág. 213241.

[9] Harvey, David. 2002. "Química Analítica Moderna". Editorial Concepción Fernández España.

[10] Ledezma M. Determinación de vitamina C en frutas por cromatografía líquida de alta resolución "HPLC". Tecnología en marcha.

[11] Skoog D.; Holler F.; Nieman T. 2001 "Principios de Análisis Instrumental". 50 ed. Editorial Mc Graw-Hill/ Interamericana de España, S.A. Madrid (España). Pág. 11-19, 937-939.

[12] Willard H.; Dean J. ; Settle F. 1991. "Métodos Instrumentales de Análisis". 5ta edición, Grupo Editorial Iberoamericana, S.A. de C.V. México D.F. (México) pág. 1 y 2.

[13] Miller J.C., Miller j.n. 1993 "Estadística para Química Analítica". 20 ed. Edición AddisonWisley Iberoamericana. Delaware (E.U.A.). pág. 42-52.

[14] Willey 2007, HPLC for Pharmaceutical Scientists, editado por YURI KAZAKEVICH y ROSARIO LOBRUTTO.

[15] Quattrocchi O. A., Abelaida S. I., Lava R. F. "Introducción a la HPLC- Aplicación y práctica", Argentina. 1992.

[16] Candelas M. G., Alanís M. G., Del Río F. "Extracción y cuantificación por HPLC de licopeno en tomate y polvo de tomate". Universidad autónoma de Guanajuato. México.

[17] Callejón R. "Desarrollo de un método de HPLC para la determinación de aminoácidos en vinagre y su validación en muestras reales" Facultad de Farmacia de la Universidad de Sevilla. España.

[18] Mostacero J., Mejía F., Gamarra O. "Taxonomía de las fanerógamas útiles del Perú”. Universidad Nacional de Trujillo 2.a ed. 2002.
[19] Meyer R, Chardonnes F, Hübner P, Lüthy J. Polymerase chain reaction (PCR) in the quality and safety assurance of food: detection of soya in processed meat products. Z Lebensm Unters Forsch A. 1996; 203: 339-344.

[20] Hupfer Ch, Hotzel H, Sachse K, Engel KH. Detection of the genetic modification in heattreated products of $\mathrm{Bt}$ maize by polymerase chain reaction. Z Lebensm Unters Forsch A 1998; 206: 203-207.

[11] Agater IB, Brianth KJ, Liewellyn JW, Sawyer R, Bailey J, Hitchcock CHS. The determination of soya and meat protein in raw and processed meat products by specific peptide analysis. $\mathrm{J}$ Sci Food Agric 1986; 37: 317-331.

[22] Janssen FW, Voortman G, De Baai JA. Detection of wheat gluten, whey protein, casein, ovalbumin, and soy protein in heated meat products by electrophoresis, blotting, and imonoperoxidase staining. J Agric Food Chem 1987; 35: 563-567.

[23] Molander E. Determination of soya protein in meat products by standard curves obtained from SDS gel electrophoresis. Z Lebensm Unters Forsch A 1982; 174: 278-281.

[24] Wang HJ, Murphy PA. Isoflavone content commercial soybean foods. J Agric Food Chem 1994; 45: 4635-4638.

[25] Murphy PA, Song T, Buseman G, Barua K. Isoflavones in soy-based infant formulas. J Agric Food Chem 1997; 42: 1666-1673.

[26] Franke AA, Custer LJ, Cerna CM, Narala KK. Quantitation of phytoestrogens in legumes by HPLC. J Agric Food Chem 1994; 9: 1905-1913.

[27] Liggins J, Bluck LJC, Coward WA, Bingham A. Extraction and quantification of daidzein and genistein in food. Anal Biochem 1998; 264: $1-7$.

[28] Wilkinson AP, Wähälä K, Williamson G. Identification and quantitation of polyphenol phytoestrogens in foods and human biological fluids. J Chromatogr B 2002; 777: 93-109.

[29] Franke AA, Custer LJ, Wang W, Yang, Shi $\mathrm{CH}$. HPLC analysis of isoflavonoids and other phenolic agents from foods and human fluids. Proc Soc Exp Biol Med 1998; 37: 263-272.

[30] Körs M, Steinhart H. CTAB electrophoresis and immunoblotting: a new method for the determination of soy proteins in meat products. Z Lebensm Unters Forsch A 1997; 205: 224-226. 
[31] Shihabi ZK, Kute T, Garcia LL, Hinsdale M. Analysis of isoflavones by capillary electrophoresis. J Chromatogr A 1994; 680: 181-185.

[32] Klejdus B, Vitamvásová D, Kubáň V. Reversedphase high-performance liquid chromatographic determination of isoflavones in plant materials after isolation by solid-phase extraction. J Chromatogr A 1999; 839: 261-263.

[33] Griffith AP, Collison MW. Improved methods for the extraction and analysis of isoflavones from soy-containing foods and nutritional supplements by reversed-phase highperformance liquid chromatography and liquid chromatography-mass spectrometry. J Chromatogr A 2001; 913: 397-413.

[34] Careri M, Elviri L, Mangia A. Validation of a highperformance liquid chromatographic method for determination of isoflavonoids in soybeans. Study of extraction procedure by experimental design. Chromatographia 2001; 54: 45-50.

[35] Hutabarat LS, Mulholland M, Greendlield H. Development and validation of an isocratic high-performance liquid chromatographic method for quantitative determination of phytoestrogens in soya bean. J Chromatogr A 1998; 795: 377-382. 